\title{
BRCA1/2 mutations and FMR1 alleles are randomly distributed: a case control study
}

Efrat Dagan, Yoram Cohen, Adi Mory, Vardit Adir, Zvi Borochowitz, Hila Raanani, Alina Kurolap, Svetlana Melikhan-Revzin, Dror Meirow and Ruth Gershoni-Baruch

European Journal of Human Genetics (2014) 22, 295; doi:10.1038/ejhg.2013.294

Correction to: European Journal of Human Genetics (2014) 22, The authors wish to acknowledge the Jack Craps Foundation for 277-279; doi:10.1038/ejhg.2013.281; published online 27 November funding their research. They would like to apologise for this omission. 2013

\section{The homozygosity index $(\mathrm{HI})$ approach reveals high allele frequency for Wilson disease in the Sardinian population}

Alessandro Gialluisi, Simona Incollu, Tommaso Pippucci, Maria Barbara Lepori, Antonietta Zappu, Georgios Loudianos and Giovanni Romeo

European Journal of Human Genetics (2014) 22, 295; doi:10.1038/ejhg.2013.261

Correction to: European Journal of Human Genetics (2013) 21, 1308-1311; doi:10.1038/ejhg.2013.43; published online 13 March 2013

Post publication, the authors of this paper realised that the authors, Tommaso Pippucci and Giovanni Romeo had been incorrectly affiliated to: Language and Genetics Department,
Max Planck Institute for Psycholinguistics, Nijmegen, The Netherlands, when they are in fact affiliated to: Unità Operativa di Genetica Medica, Dipartimento di Scienze Mediche e Chirurgiche Policlinico Sant' Orsola-Malpighi, Bologna, Italy.

The authors would like to apologise for their error. 